\title{
Prophylaxis of Pneumocystis carinii pneumonia: too much of a good thing?
}

\author{
Robert F Miller \\ Windeyer Institute of Medical Sciences, Royal Free and University College Medical \\ School, University College London, and Camden and Islington Community Health \\ Services (NHS) Trust, Mortimer Market Centre, London WC1E 6AU, UK
}

Introductory articles

\section{Efficacy of azithromycin in prevention of Pneumocystis carinii pneumonia: a randomised trial}

MW Dunne, S Bozzette, JA McCutchan, MP Dubé, FR Sattler, D Forthal, CA Kemper, D Havlir, for the California Collaborative Treatment Group

Background. Azithromycin in combination with sulphonamides is active against Pneumocystis carinii $(P C P)$ in animals. We assessed the clinical efficacy of azithromycin for PCP prophylaxis in human beings. Methods. We identified HIV-1-infected patients with PCP during a prospective randomised trial comparing azithromycin, rifabutin, and the two drugs in combination for prevention of disseminated? Mycobacterium avium infection. Patients had CD4-cell counts less than 100/ $\mathrm{L}$ at entry and received PCP prophylaxis according to the standard practice of their clinician. Analysis was by intention to treat. Findings. Patients receiving azithromycin, either alone $(n=233)$ or in combination with rifabutin $(n=$ 224), had a 45\% lower risk of developing PCP than those receiving rifabutin alone $(n=236 ; p=\mathbb{\varnothing}$ 0.008). Compared with rifabutin alone, hazard ratio for azithromycin was 0.54 (95\% Cl 0.32-0.94), for $\overrightarrow{\vec{B}}$ azithromycin plus rifabutin was $0.55(0.32-0.94)$, and for regimens containing azithromycin was 0.553 (0.35-0.86). The most common side-effects involved the gastrointestinal tract with dose-limiting 㞔 toxicities, and were mainly seen in patients receiving combination therapy. Interpretation. Azithromycin as prophylaxis for $\mathrm{M}$ avium complex disease provides additional protection against $\mathrm{P}$ carinii over and above that of standard PCP prophylaxis. Use of azithromycin is beneficial only as primary prophylaxis. (Lancet 1999;354:891-5)

Discontinuation of Pneumocystis carinii pneumonia prophylaxis after start of highly active antiretroviral therapy in HIV-1 infection

GJ Weverling, A Mocroft, B Ledergerber, O Kirk, J Gonzáles-Lahoz, A d'Arminio Monforte, R Proenca, AN Phillips, JD Lundgren, P Reiss, for the EuroSIDA Study Group

Background. Highly active antiretroviral therapy (HAART) has improved rates of CD4-lymphocyte recovery and decreased the incidence of HIV-1-related morbidity and mortality. We assessed whether prophylaxis against Pneumocystis carinii pneumonia (PCP) can be safely discontinued after HAART is started. Methods. We investigated 7333 HIV-1-infected patients already enrolled in EuroSIDA, a continuing prospective observational cohort study in 52 centres across Europe and Israel. We did a $\frac{0}{0}$ person-years analysis of the rate of discontinuation of PCP prophylaxis and of the incidence of PCP after $\approx$ the introduction of HAART into clinical practice from July, 1996. Findings. The rate of discontinuation of $\overrightarrow{\mathbb{Q}}$ primary and secondary PCP prophylaxis increased up to 21.9 discontinuations per 100 person-years of 2 follow-up after March, 1998. 378 patients discontinued primary (319) or secondary (59) prophylaxis a 8 median of 10 months after starting HAART. At discontinuation for primary and secondary prophylaxis, respectively, the median CD4-lymphocyte counts were $274 \mathrm{cells} / \mu \mathrm{L}$ and $270 \mathrm{cells} / \mu \mathrm{L}$, the median plasma

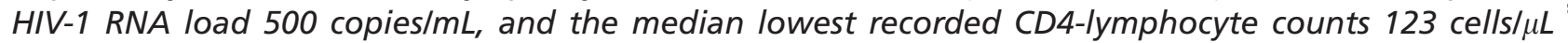
and $60 \mathrm{cells} / \mu \mathrm{L}$. During 247 person-years of follow-up, no patient developed PCP (incidence density 0 [95\% Cl 0-1.5]). Interpretation. The risk of PCP after stopping primary prophylaxis, especially in patients on HAART with a rise in CD4-lymphocyte count to more than 200 cells/ $\mu \mathrm{L}$, is sufficiently low to warrant 
discontinuation of primary PCP prophylaxis. Longer follow-up is needed to confirm a similarly low risk for stopping secondary PCP prophylaxis. (Lancet 1999;353:1293-8)

\title{
Discontinuation of primary prophylaxis against Pneumocystis carinii pneumonia in HIV-1-infected adults treated with combination antiretroviral therapy
}

\author{
H Furrer, M Egger, M Opravil, E Bernasconi, B Hirschel, M Battegay, A Telenti, PL Vernazza, \\ M Rickenbach, M Flepp, R Malinverni, for the Swiss HIV Cohort Study
}

Background. It is unclear whether primary prophylaxis against Pneumocystis carinii pneumonia can be discontinued in patients infected with the human immunodeficiency virus (HIV) who are successfully? treated with combination antiretroviral therapy. We prospectively studied the safety of stopping prophylaxis among patients in the Swiss HIV Cohort Study. Methods. Patients were eligible for our study if their CD4 counts had increased to at least 200 cells per cubic millimeter and 14 percent of total lymphocytes while they were receiving combination antiretroviral therapy, with these levels sustained $\underset{\otimes}{*}$ for at least 12 weeks. Prophylaxis was stopped at study entry, and patients were examined every three of months thereafter. The development of P. carinii pneumonia was the primary end point, and the $\stackrel{\text { in }}{5}$ development of toxoplasmic encephalitis the secondary end point. Results. Of the 262 patients included $\overline{0}$ in our analysis, 121 (46.2 percent) were positive for IgG antibodies to Toxoplasma gondii at base line. $\vec{f}$ The median CD4 count at study entry was 325 per cubic millimeter (range, 210 to 806); the median os nadir CD4 count was 110 per cubic millimeter (range, 0 to 240). During a median follow-up of 11.3 o months (range, 3.0 to 18.8), prophylaxis was resumed in nine patients, and two patients died. There 3 were no cases of P. carinii pneumonia or toxoplasmic encephalitis. The one-sided upper 99 percent $?$ confidence limit for the incidence of P. carinii pneumonia was 1.9 cases per 100 patient-years (based $\stackrel{0}{0}$ on 238 patient-years of follow-up). The corresponding figure for toxoplasmic encephalitis was 4.2 per $\frac{0}{N}$ 100 patient-years (based on 110 patient-years of follow-up). Conclusions. Stopping primary prophylaxis 8 against P. carinii pneumonia appears to be safe in HIV-infected patients who are receiving combination antiretroviral treatment and who have had a sustained increase in their CD4 counts to at least 200 cells per cubic millimeter and to at least 14 percent of total lymphocytes. (N Engl J Med 1999;340:1301-6)

The fungal pathogen Pneumocystis carinii was first described by Chagas in 1909 but its clinical importance was not recognised until 1951when it was identified as the cause of interstitial plasma cell pneumonia, which had been described in Europe in the late 1930s and 1940 s in premature and malnourished children. In the 1960s $P$ carinii pneumonia occurred mainly in children with congenital defects of the immune system and in both adults and children with acquired immune defects due to malignancy or its treatment. 2 With the advent of organ transplantation, it became clear that $P$ carinii pneumonia was associated with the immunosuppression used to prevent organ rejection. With prophylaxis, case rates fell in these populations. In 1980 P carinii pneumonia was the first opportunistic infection to be associated with HIV infection, and the AIDS pandemic was defined. ${ }^{3}$.

\section{Who gets $P$ carinii pneumonia?}

Most cases of $P$ carinii pneumonia occur in patients with abnormalities of $\mathrm{T}$ lymphocyte function or numbers: $P$ carinii pneumonia rarely occurs in patients with isolated $\mathrm{B}$ cell defects. Before the introduction of prophylaxis, attack rates for $P$ carinii pneumonia varied from $22-43 \%$ in children with acute lymphoblastic leukaemiat and $27-42 \%$ in children with severe combined immunofeficiency, to $25 \%$ in those with rhabdomyosarcoma In patients who do not receive prophylaxis after organ transplantation, attack rates for $P$ carinii pneumonia vary from $4-10 \%$ following renal transplantation to $16-43 \%$ following heart or heart/lung

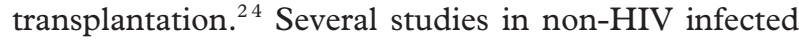

patients have suggested, regardless of the nature of the $\overline{\bar{O}}$ underlying immunosuppression, that glucocorticoids are an independent risk factor for development of $P$ 우 carinii pneumonia|

In HIV infected patients the CD4 $+\mathrm{T}$ lymphocyte 음 count is used in clinical practice both to determine the risk of $P$ carinii pneumonia in an individual patient $\bar{\sigma}$ and to make recommendations about when to start $\stackrel{3}{3}$ prophylaxis (see below) ${ }^{6-10}$ There is increasing evidence $\frac{\delta}{3}$ that the CD $4+\mathrm{T}$ lymphocyte count may also be useful

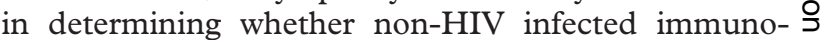
suppressed jndividuals are at risk of $P$ carinii $\frac{D}{0}$ pneumonia ${ }^{1}||^{2}$

Who should receive prophylaxis?

The United States Public Health Service and the Infectious Diseases Society of America have made the following recommendations.

HIV infected patients, including those who are preg- $\stackrel{?}{+}$ nant and those receiving combination antiretroviral therapy (also known as highly active antiretroviral therapy $\underset{\mathbb{D}}{\mathrm{D}}$ or HAART), should receive primary prophylaxis against $\frac{\mathcal{P}}{\mathbb{D}}$ $P$ carinii pneumonia if they have a CD $4+\mathrm{T}$ lymphocyte $\varrho$ count of $<200$ cells/ $\mu$ l or a history of oral/pharyngeal 2 candidiasis ${ }^{10}$ Prophylaxis should also be given to 8 patients who have a CD $4+\mathrm{T}$ lymphocyte count which is $<14 \%$ of the total lymphocyte count or a history of an AIDS defining illness - for example, Kaposi's sarcoma ${ }^{10}$ If it is not possible to monitor the CD4+ $\mathrm{T}$ lymphocyte count frequently (at least every three months), then consideration should be given to starting prophylaxis in patients whose CD $4+\mathrm{T}$ lymphocyte 
counts are between 200 and 250 cells/ $\mu 1 \|\left.^{1}\right|^{13}$ Secondary prophylaxis should be given to all HIV infected patients who have had an episode of $P$ carinii pneumonia, regardless of their CD4 $+\mathrm{T}$ lymphocyte count. Although HAART may cause a rise in the CD $4+\mathrm{T}$ lymphocyte count to $>200$ cells $/ \mu$, there are insufficient data to recommend stopping secondary prophylaxis against $P$ carinii pneumonia (see below). ${ }^{10}$

In the non-HIV infected immunosuppressed population, those with high attack rates for $P$ carinii pneumonia should receive prophylaxis ${ }^{2}$ Sepkowitz et al have suggested that, in addition, patients with any immune dysfunction induced by an inflammatory disease or by radiotherapy or chemotherapy who receive $20 \mathrm{mg}$ /day of prednisolgne (or equivalent) should receive prophylaxis 14.

What drug regimen is preferred for prophylaxis? Co-trimoxazole $960 \mathrm{mg}$ once daily is the regimen of choice for prophylaxis of $P$ carinii pneumonia in both HIV infected and non-HIV infected immunosuppressed individuals. 11 Alternative agents which, in HIV infected patients, have been shown to be less effective include dapsone (with or without pyrimethamine), ${ }^{1}$ nebulised pentamidine ${ }^{17}$ and atovaquone ${ }^{18}$

The ideal agent for prophylaxis is cheap, readily available, effective, easily administered so that compliance is maintained, has low toxicity, and does not interact adversely with HAART or chemotherapy/ immunosuppressive drug regimens. An additional benefit would be for it to provide "cross prophylaxis" against other micro-organisms. Cross prophylaxis is demonstrated clearly for co-trimoxazole at a dose of $960 \mathrm{mg}$ once daily. In addition to effectively preventing $P$ carinii pneumonia, co-trimoxazele also protects against cerebral toxoplasmosi.1. ${ }^{19}$ and some bacterial infections 1 . $^{20}$ Lower doses of co-trimoxazole such as $480 \mathrm{mg}$ once dail ${ }^{21}$ or $960 \mathrm{mg}$ three times a wee ${ }^{22}$ are also effective regimens for prophylaxis and may also confer cross prophylaxis. Immunosuppressed HIV noninfected patients with antibodies to Toxoplasma gondi should not receive nebulised pentamidine as prophylaxis because of the risk of development of cerebral toxoplasmosis. 10

\section{What are the long term benefits of prophylaxis?}

The widespread availability and uptake of co-trimoxazole and other agents as prophylaxis has had a major impact on the incidence of $P$ carinii pneumonia. In one study in London $P$ carinii pneumonia accounted for $23 \%$ of all admissions to a specialist HIV unit in 1986-7 (before prophylaxis was used) but was responsible for only $13 \%$ of admissions in 1990-1 (by which time prophylaxis had been introduced) ${ }^{23}$ Using data from 90 HIV centres in the USA, the Centres for Disease Control and Prevention, Atlanta found a reduction in the incidence of $P$ carinii pneumonia from 9.0 per 100 person years in 1991 to 5.3 per 100 person years in 1996. ${ }^{24}$ Prophylaxis has also had an impact on survival time in patients with HIV infection and CD4+ $\mathrm{T}$ lymphocyte counts of $<200$ cells $/ \mu 1$. In a study from San Francisco the median survival increased from 28.4 months in patients studied between 1983 and 1986 to 38.1 months in those studied between 1988 and $1993 .{ }^{2}$ The largest increase in survival time was seen in those who had $P$ carinii pneumonia, suggesting that prophylaxis and treatment of this infection were important factors in longer survival ${ }^{25}$ It is important to remember that these benefits from prophylaxis were reported before the advent of HAART.

What are the costs of prophylaxis?

The cost of prophylaxis varies considerably from $£ 20$ (US\$60) per year for co-trimoxazole to £6346 (US $\$ 10647$ ) per year for atovaquone.

\section{What adverse events are associated with prophylaxis?}

Adverse reactions to co-trimoxazole, consisting of rash and/or fever, occur more commonly in patients immunosuppressed by HIV infection than in those immunosuppressed by other causes or those with normal immunity ${ }^{26}$ An explanation for this difference is not $x$ apparent. Hypotheses include HIV induced immuno- of pathogenic effects or changes in acetylator dependent $\frac{\text { D }}{0}$ metabolism of co-trimoxazole to toxic (hydroxylamine) 으 metabolites ${ }^{27}$ Desensitisation to co-trimoxazole should $\overrightarrow{-}$ be attempted before considering a change to alternative, less effective prophylaxis ${ }^{2} \mid 29$

\section{What effect does long term prophylaxis have on other pathogens?}

As co-trimoxazole is a potent broad spectrum antibacterial agent in addition to being the most effective 8 agent for prophylaxis of $P$ carinii pneumonia, concerns have been expressed that its widespread long term use may lead to selection of resistance in bacteria. Until recently, once prophylaxis with co-trimoxazole was started, it was recommended that it should be used for $\stackrel{0}{0}$ the rest of the HIV infected person's life. It has been $\stackrel{\mathbb{Q}}{\square}$ suggested that resistant bacteria might develop in these $\overrightarrow{\overrightarrow{0}}$ individuals and subsequently spread into the general 3 population. In the non-HIV infected population there is already evidence that co-trimoxazole, used as prophylaxis in patients with acute leukaemia, may ipduce plasmid type resistance in Enterobacteriaceae ${ }^{30}$ In patients with HIV infection the use of co-trimoxazole has been associated with the development of co-trimoxazole resistant Streptococcus pneumoniat. ${ }^{32}$

A serial cross sectional study from San Francisco General Hospital, USA has examined rates of resistance to co-trimoxazole among isolates of Staphylococcus aureus and also among seven common genera of the Enterobacteriaceae (including Escherichia coli and Klebsiella spp) from HW infected and non-infected inpatients and 5 outpatients ${ }^{33}$ The study period was from 1988 until $N$ 1995. At the start of the study period co-trimoxazole $\tilde{\omega}^{N}$ was not commonly used as nebulised pentamidine was the preferred option for prophylaxis, but co-trimoxazole 0 subsequently became first choice for prophylaxis. Overall resistance rates (in all eight genera combined) were $\stackrel{?}{?}$ between $2.8 \%$ and $5.5 \%$ before 1988 and have risen 7 steadily from $7.2 \%$ in 1988 to $20.4 \%$ in 1995.33 This $\frac{0}{\mathbb{D}}$ trend was most apparent among HIV infected patients in whom overall resistance increased from $6.3 \%$ in $1988 \Omega$ to $53 \%$ in 1995 . The largest increases in resistance were in isolates of $S$ aureus (from $0 \%$ in 1988 to $48 \%$ in 8 1995 ) and $E$ coli (from $24 \%$ to $74 \%$ ) from HIV infected patients. These data have implications for the clinician, 흘 as the long term benefit of co-trimoxazole needs to be ?+? balanced against its effect on resistance. Despite the limitations of this study (it did not control for possible influences of concomitant therapies and the study groups were incompletely defined), its findings have 
led to a reduction in the use of co-trimoxazole for prophylaxis and treatment of infections other than $P$ carinii pneumonia at San Francisco General Hospita. 33

\section{Can $\boldsymbol{P}$ carinii become resistant to prophylaxis?}

Conventional methods of determining drug resistance cannot be used to answer this question as human derived $P$ carinii cannot be cultured reliably in vitro. The drug target for sulphamethoxazole (and for dapsone, which is a sulphone) is dihydropterate synthase (DHPS) and for trimethoprim is dihydrofolate reductase (DHFR); these are sequential enzymes in the metabolism of folate. In several bacteria, including E coli, Neisseria meningitidis, and $S$ pneumoniae, and in the protozoan Plasmodium falciparum, point mutations within conserved regiops of the DHPS gene confer resistance to sulphonamides. ${ }^{3-36}$ In $P$ carinii DHPS is part of a trifunctional protein, along with dihydroneopterin aldolase and hydroxymethyldihydropterin pyrophosphokinase - two other enzymes in the folic acid metabolic pathway ${ }^{3}$

The first evidence of mutations in the DHPS gene of $P$ cariniz came from case report ${ }^{38}$ and a study of 27 patients 39 Development of resistance was suggested by the finding of non-synonymous single base pair polymorphisms (point mutations) within a highly conserved region of the DHPS gene of $P$ carinii resulting in amino acid substitutions at codons 55 and $57 .{ }^{39}$ These amino acid substitutions are at or near to the sulpha binding sites and are homologous to mutations which cause resistance to sulpha drugs in other pathogens such as $E$ coll ${ }^{40}$ Mutations in the $P$ carinii DHPS gene were identified in seven of 27 patients and were more commonly seen in HIV infected patients with $P$ carinii pneumonia who had failed co-trimoxazole or dapsone prophylaxis ${ }^{39}$ The common "wild type" DHPS of $P$ carinii contains threonine at codon 55 and proline at codon 57. Single mutations are seen with threonine $\rightarrow$ alanine (codon 55) and proline $\rightarrow$ serine (codon 57). A "double mutant" exists when both amino acid substitutions occur in the same sample.

Although strongly suggestive of sulpha resistance, these data do not prove that the sequence polymorphisms confer drug resistance ${ }^{40}$ Stronger evidence comes from Mei et al who reported two patients who developed P carinii pneumonia while on co-trimoxazole prophylaxis ${ }^{39}$ Treatment with high dose co-trimoxazole was ineffective in one and this patient recovered when treatment was changed to pentamidine. The other patient, despite recovery with co-trimoxazole, had two further episodes of $P$ carinii pneumnnia. Both patients had "double mutant" DHPS genes ${ }^{39}$

Helweg-Larsen et al studied bronchoscopic alveolar lavage samples from 152 episodes of $P$ carinii pneumonia in 144 patients from a prospective cohort in Copenhagen, Denmark and assessed, firstly, whether mutations in the DHPS gene of $P$ carinii were associated with prior exposure to sulpha drugs and, secondly, whether DHPS mutations influenced outcome from $P$ carinii pneumonia. ${ }^{41}$ A portion of the DHPS gene was analysed using PCR and sequencing; "wild type" $P$ carinii DHPS was found in 121 episodes $(79.6 \%)$ and DHPS mutations in 31 episodes $(20.4 \%)$ the three month survival was significantly lower in patients with $P$ carinii pneumonia and DHPS containing mutations than in patients with "wild type" DHPS. Factors such as low CD $4+\mathrm{T}$ lymphocyte count, hypoxaemia, and older age have all been shown to be associated with a poor outcome from $P$ carinii pneumonia. Even after adjustment for these prognostic variables, patients with "mutant" DHPS P carinii pneumonia were three times more likely to die than those with "wild type" (hazard ratio $=3.1 ; 95 \%$ CI 1.2 to 8.1 ) ${ }^{41}$

Of note, DHPS mutations were not always associated with a failure to respond to co-trimoxazole as high dose co-trimoxazole was successful in 12 of 19 episodes $\overrightarrow{\vec{c}}$ with "mutant" DHPS 41 This observation suggests that $\frac{7}{0}$ strains of $P$ carinii with only one or two DHPS mutations $\frac{\square}{\sigma}$ are only partially resistant to sulpha drugs. ${ }^{40}$ In $E$ coli $\overline{\text { के }}$ mutations in DHPS are associated with a low level $\mathbb{D}$ of resistance to sulpha drugs; as additional mutations accumulate, high level resistance develops ${ }^{42}$ It is conceivable that the same phenomena may be occurring in $P$ carinii.

In contrast, there appears to be no evidence for $\stackrel{\omega}{S}$ development of mutations in the DHFR gene of $P \overrightarrow{5}$ carinii in natients who have received co-trimoxazole or dapsone ${ }^{43}$ In a recent study from the National Institutes of of Health, Bethesda, USA the DHFR gene of human on derived $P$ carinii was cloned and sequenced, and evi- $\stackrel{\infty}{=}$ dence of both DHPS and DHFR mutations yas sought in 37 isolates of $P$ carinii from 35 patients ${ }^{43}$ Although $\rightarrow$ 15 patients had previously received trimethoprim (in co- is trimoxazole), all isolates had identical DHFR sequences $G$ apart from one isolate which had a synonymous poly- $\frac{O}{}$ morphism (which did not result in a substitution of the $\vec{\nabla}$ derived amino acid). By contrast, 16 of 37 samples had non-synonymous DHPS polymorphisms ("mutant" type DHPS) which was associated with prior use of co- $\frac{\vec{N}}{\mathrm{~N}}$ trimoxazole/dapsone prophylaxis. ${ }^{3}$ These results sug- 8 gest that there is less selective pressure from drug exposure on DHFR than on DHPS. Although cotrimoxazole is routinely given to patients, there is little evidence from animal models of $P$ carinii pneumonia that the trimethoprim-cemponent adds to the efficacy of sulphamethoxazole $4{ }^{4}{ }^{5}$

The drug target of atovaquone is the ubiquinone $\overrightarrow{\vec{A}}$ binding sites in cytochrome $\mathrm{b}$ of $P$ carinii. Compared 3 with co-trimoxazole, atovaquone is used much less commonly for the prophylaxis and treatment of $P$ carinii pneumonia; however, non-synonymous sequence polymorphisms have been identified in the cytochrome $b \frac{0}{2}$ gene of $P$ carinii from patients with prior exposure to atovaquone ${ }^{46}$ This suggests that $P$ carinii may become resistant to other drugs.

A strategy of reducing or preventing the emergence of sulpha resistance in $P$ carinii by using other agents 0 for prophylaxis ("drug switching") is hampered by the ? availability of other equally effective drugs. The impact $\frac{7}{2}$ of HAART (see below) will certainly reduce the size of

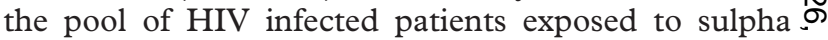
drugs for long periods, but this class of drug will continue $N$ to be used widely in those patients immunosuppressed N by other causes ${ }^{2}$ Prevention of development of atovaquone resistance in $P$ falciparum is achieved by combining it with proguanil ${ }^{46}$ Perhaps, if atovaquone is $\bar{D}$ used for prophylaxis of $P$ carinii pneumonia, it should $\stackrel{?}{+}$ be combined with another drug such as pyrimethamine to hinder development of resistance. The sordarins $\frac{\vec{D}}{\mathbb{D}}$ which target fungal translation-elongation factor 2 and $\cong$ inhibit fungal protein synthesis are active in vitro against $\stackrel{\varrho}{\circ}$ a range of fung ${ }^{47}$ and in the animal model of $P$ carinii 2 pneumonia. As yet, the efficacy of sordarins in human 8 $P$ carinii pneumonia has not been studied.

\section{Does azithromycin have activity against $P$ carinit?}

Animal data suggest that macrolide antibiotics, in addition to activity against bacteria and mycobacteria, also 
have activity against $P$ carinii. Studies using the animal model of $P$ carinii pneumonia (the dexamethasone immunosuppressed rat) also show that combinations of sulphonamide and macrolides (erythromycin, clarithromycin and azithromycin) are active against $P$ carinii and that these combinations are possibly synergistic ${ }^{48}$

In the first introductory article by Dunne et a $4^{49}$ azithromycin given in a dose of $1200 \mathrm{mg}$ once weekly, with or without rifabutin $300 \mathrm{mg}$ daily, was superior to rifabutin alone when given for prophylaxis of disseminated Mycobacterium avium complex (dMAC) in a double blind randomised trial of 693 patients with HIV infection and median CD4 $+\mathrm{T}$ lymphocyte counts of $<40$ cells $/ \mu$ l. Although the study was powered to show a difference between treated groups in prevention of dMAC, it also had $80 \%$ power to detect a $33 \%$ reduction in $P$ carinii pneumonia at a significance level of 0.05 . Overall, in this study 185 patients $(26.7 \%)$ had a previous episode of $P$ carinii pneumonia at entry. All patients continued their $P$ carinii prophylaxis (co-trimoxazole $58.7 \%$, dapsone $18.6 \%$, pentamidine $17 \%$, combinations of drugs $3.3 \%$, none $2.6 \%$ ) in addition to receiving azithromycin alone (233 patients) or with rifabutin (224 patients) or rifabutin alone (236 patients). Median follow up was 514 days and 78 patients had 85 episodes of $P$ carinii pneumonia, 48 of which (56\%) were first episodes. Twice as many episodes of $P$ carinii pneumonia per 100 patient years were reported in patients receiving rifabutin alone than in those receiving azithromycin, with or without rifabutin $(p=0.008) .{ }^{49}$ Two important observations arise from this study. Firstly, the benefit of azithromycin in providing additional protection against $P$ carinii pneumonia was seen only in the 508 patients who were taking primary prophylaxis and not in the 185 with a past history of $P$ carinii pneumonia - that is, those receiving secondary prophylaxis. However, the numbers of patients in this subgroup were small so there was insufficient power to detect whether there was a true difference. Secondly, the lowest rates of $P$ carinii pneumonia were seen in those who received prophylaxis with co-trimoxazole or dapsone. 49

Interpretation of the study data suggests a possible synergism between azithromycin and sulpha drugs. However, activity from azithromycin alone was demonstrated as, in the patients who did not receive cotrimoxazole or dapsone, fewer cases of $P$ carinii pneumonia were seen in those who received azithromycin than in those who received rifabutin 49 The authors hypothesise that an alternative explanation for their observations might be that rifabutin in fact increases the risk of $P$ carinii pneumonia, although this suggestion is not borne out by their data as the incidence (attack rate) of $P$ carinii pneumonia was similar in those receiving azithromycin alone and azithromycin with rifabutin. A drug-drug interaction remains a possible explanation. Although no experimental data exist for a drug-drug interaction between rifabutin and sulphamethoxazole or dapsone, there is a significant interaction between rifampicin and dapsone with the former drug producing a $7-10$ fold reduction in dapsone levels, rendering it ineffective $55^{51}$

\section{What is the impact of HAART?}

Several recent studies have shown that the introduction of HAART has had a significant impact on the prognosis of patients with HIV infection. These studies have shown reductions in the incidence of several op- portunistic infections - including $P$ carinii pneumonia, $\mathrm{dMAC}$, and cytomegalovirus retinitis - admissions to hospital, and overall mortality from HIV infection. $55^{5}$ These benefits have occurred over and above that gained by prophylaxis of specific opportunistic infections alone. During the time of these studies the marked reductions $\overrightarrow{\overrightarrow{\mathrm{S}}}$ in the incidence of opportunistic infections occurred when there was no major change in uptake of prophylaxis $\frac{\bar{\sigma}}{\sigma}$ by patients, nor were there changes to the US Public $\overline{\bar{p}}$ Health Service/Infectious Diseases Society of America $\overparen{\nabla}$ guidelines for the use of prophylaxis to prevent on portunistic infections in patients with HIV infection 54 os HAART brings about a rapid decrease in plasma HIV $\vec{\circ}$ RNA and an increase in peripheral blood CD4 $+\mathrm{T} \overrightarrow{\vec{\omega}}$ lymphocyte counts within a few weeks in most patients. The resulting "partial immune reconstitution" is shown $\overrightarrow{\overrightarrow{5}}$ initially by a largely clonal expansion of memory CD $4+\frac{9}{8}$ T lymphocytes. Subsequently there is death of activated of $\mathrm{CD} 4+\mathrm{T}$ lymphocytes and increases in naive CD $4+$ on $\mathrm{T}$ lymphocytes are seen with an expansion in the di- $\frac{\infty}{0}$ versity of the $\mathrm{T}$ lymphocyte repertoire. It is unclear just how much CD $4+\mathrm{T}$ lymphocyte function is restored by $\overrightarrow{.}$ HAART induced partial immune reconstitution. Some os researchers report a return to normal of cell mediated $\mathcal{G}$ immunity against opportunistic pathogens such as cyto- 을 megalovirus and Mycobacterium tuberculosis within 3-6 $\vec{\longrightarrow}$ months of commencing HAART.5 5

The observation that HAART gives additional pro- $\frac{0}{\infty}$ tection over and above that derived from specific pro- $\frac{2}{N}$ phylaxis for $P$ carinii led several groups to question 8 whether prophylaxis could be safely discontinued in patients who had HAART induced partial immune reconstitution. Results from four observational cohort studies (including the introductory articles by Weverling et $a{ }^{58}$ and Furrer et $a{ }^{59}$ and one retrospective case $\stackrel{2}{8}$ control study provide evidence to support stopping $\stackrel{\mathbb{Q}}{\varrho}$ primary $P$ carinii prophylaxis in HIV infected patients $\overrightarrow{\overrightarrow{0}}$ who have had partial immune reconstitution with 3 HAART. ${ }^{8}-62$

The study by Weverling et $\sqrt{2}$ was carried out on 7333 HIV infected patients from 52 centres in Europe and Israel who were participating in the EUROSIDA study. The study aimed to describe the incidence of $P$. carinii pneumonia within the EUROSIDA cohort and 3 to see how this had changed with time; it also aimed to describe the incidence of $P$ carinii pneumonia following 3 the widespread introduction of HAART after July 1996 O in those who discontinued $P$ carinii prophylaxis. ${ }^{5}$ The overall incidence of $P$ carinii pneumonia fell from $49 \frac{T}{O}$ cases per 100 person years of follow up before March 1995 to 0.3 cases per 100 person years after March 5 1998. Three hundred and seventy eight patients dis- $N$ continued $P$ carinii prophylaxis after starting HAART; స్ $319(84 \%)$ had received primary prophylaxis and 59 응 $(16 \%)$ were receiving secondary prophylaxis. The me- 0 dian CD $4+$ T lymphocyte count at the time prophylaxis was discontinued was 274 cells/ $\mu \mathrm{l}$ (primary) and $270 ?$ cells $/ \mu$ l (secondary). Overall, the median HIV-1 RNA load was 500 copies $/ \mathrm{ml}$ at the time prophylaxis was discontinued. However, 98 patients $(26 \%)$ discontinued $P$ carinii prophylaxis with CD $4+$ T lymphocyte counts of $<200$ cells/ $\mu$ l and a median HIV-1 RNA load of 3311 울 copies $/ \mathrm{ml}$. In those with CD $4+\mathrm{T}$ lymphocyte counts 8 of $<200$ cells $/ \mu$ and an HIV-1 RNA load of $<500$ 을 copies $/ \mathrm{ml}$ at the time prophylaxis was discontinued, the 흘 withdrawal of prophylaxis occurred a median of eight $\stackrel{?}{?}$ months (primary) and five months (secondary) after starting HAART ${ }^{58}$ No cases of $P$ carinii pneumonia were seen during 247 person years of follow up, irrespective of the last CD $4+$ T lymphocyte count/HIV-1 RNA load 
at the time of stopping $P$ carinii prophylaxis, the lowest ever (nadir) CD $4+$ T lymphocyte count before HAART was started, or the duration of HAART. Of note, there were also no cases of cerebral toxoplasmosis and only one case of recurrent bacterial pneumonia was seen, in a patient with a CD $4+\mathrm{T}$ lymphocyte count of $>300$ cells $/ \mu 1{ }^{58}$

The study by Furrer et al from the Swiss HIV cohort included patients from seven treatment centres.59 Two hundred and sixty three patients receiving $P$ carinii prophylaxis who had sustained increases in CD4 $+\mathrm{T}$ lymphocyte counts to $>200$ cells $/ \mu 1$ or a CD $4+$ :total lymphocyte ratio of $>14 \%$ for three months or more after starting HAART were included in the study. The primary end point was development of $P$ carinii pneumonia and the secondary end point was development of cerebral toxoplasmosis. The median CD4 $+\mathrm{T}$ lymphocyte count at entry was 325 cells $/ \mu 1$ and the median nadir count had been 110 cells/ $\mu$. No $P$ carinii pneumonia or cerebral toxoplasmosis occurred during the follow up period (median 11.3 months, range 3.0 -18.8). Nine patients resumed prophylactic treatment, because the CD $4+\mathrm{T}$ lymphocyte count fell to $<200$ cells $/ \mu 1$ in seven cases, because of recurrent bacterial chest infection in one, and in one for personal reasons.

The study by Schneider et $\mathrm{l}^{60}$ from Utrecht, Netherlands examined the incidence of $P$ carinii pneumonia after discontinuation of prophylaxis (both primary and secondary) if CD4 + T lymphocyte counts were $>200$ cells/ $\mu 1$ documented on two occasions at least one month apart after starting HAART. The study end point was development of $P$ carinii pneumonia. Seventy eight patients discontinued prophylaxis (primary $=62$ patients) at which time their median CD $4+\mathrm{T}$ lymphocyte count was 347 cells $/ \mu l$. Their median nadir count before starting HAART was 79 cells/ $\mu$ l. HIV-1 RNA was not detectable in 61 patients and was 15000 copies/ $\mathrm{ml}$ in the other 17. Prophylaxis was discontinued at a mean (SD) of 9.8 (6.4) months after starting HAART. No patients developed $P$ carinii pneumonia during mean (SD) follow up of 12.7 (7.8) months ${ }^{60}$

In December 1997 guidelines for discontinuing prophylaxis were changed at four major AIDS centres in Denmark. Kirk et al studied 219 consecutive patients who stopped $P$ carinii prophylaxis (primary prophylaxis in 193 patients) when their CD4 + T lymphocyte counts rose above 200 cells 41 for more than six months in response to HAART. ${ }^{61}$ As a group, the mean nadir CD4 + T lymphocyte had been 117 cells/ $\mu$ l (primary prophylaxis) and $<50$ cells/ $\mu \mathrm{l}$ (secondary prophylaxis). One person developed $P$ carinii pneumonia after 174 person years of follow up, an overall incidence of $P$ carinii pneumonia of 0.6 cases per 100 person years
(95\% CI 0.0 to 3.2) ${ }^{61}$ This patient had been on HAART for 1.7 years and had stopped co-trimoxazole prophylaxis five months previously. At this time the CD4 + $T$ lymphocyte count had been 216 cells/ $\mu$ l and the HIV-1 RNA load was 40000 cells $/ \mu 1$. The CD $4+\mathrm{T}$. lymphocyte count had fallen to 143 cells/ $\mu$ at the time of $\overrightarrow{\vec{*}}$ presentation with $P$ carinii pneumonia. For two months $\overrightarrow{0}$ before developing $P$ carinii pneumonia this patient had $\frac{C}{0}$ received G-CSF. The anti-inflammatory effects of G- $\overline{\bar{p}}$ CSF may well have contributed to the occurrence of $P \frac{\mathbb{Q}}{\Omega}$ carinii pneumonia in this case ${ }^{6}$

Rodriguez-Guardado et al retrospectively compared o HIV infected patients who had evidence of HAART $\vec{\circ}$ induced partial immune reconstitution with rises in $\overrightarrow{\vec{\omega}}$ CD $4+$ T lymphocyte counts to $>200$ cells $/ \mu \sqrt{62}$ Twenty ${ }^{\circ}$ six patients discontinued prophylaxis against $P$ carinii $\overrightarrow{\vec{\sigma}}$ pneumonia and 27 continued prophylaxis. The two groups were similar in terms of HIV risk factor, type of $\dot{x}$ prophylaxis and regimens of HAART, and mean CD $4+$ of T lymphocyte count. In those who stopped prophylaxis $\frac{\text { }}{0}$ the mean CD $4+\mathrm{T}$ lymphocyte count was 310 (range 음 216-486) cells $/ \mu$ l. Cases were followed for a mean of $\rightarrow$ 291 days; no cases of $P$ carinii pneumonia were seen. $\mathcal{C}$

On the basis of these data the United States Public $\mathcal{G}$ Health Service/Infectious Diseases Society of America now recommend that primary prophylaxis can be safely $\vec{\overrightarrow{ }}$ discontinued in patients responding to HAART with $\stackrel{D}{\llcorner}$ sustained increases in $\mathrm{CD} 4+\mathrm{T}$ lymphocyte counts $\frac{0}{\omega}$ above 200 cells $/ \mu{ }^{10}$ The guidelines acknowledge, firstly, $\frac{\text { ? }}{N}$ that data to support the recommendations come largely 8 from patients who began HAART which included a protease inhibitor, secondly, that the median follow up was 6-12 months and the median CD $4+\mathrm{T}$ lymphocyte count at the time prophylaxis was discontinued was $>300$ cells/ $\mu$ l and, thirdly, that many of the patients who discontinued prophylaxis had a sustained suppression $\stackrel{\mathbb{Q}}{\varrho}$ of $\mathrm{HIY}-1$ RNA load to below detection limits of available $\overrightarrow{\overrightarrow{0}}$ assays. ${ }^{10}$

What if, despite HAART, CD4 $+\mathrm{T}$ lymphocyte counts fall below 200 cells/ $\mu$ l and/or HIV-1 RNA increases again? Current recommendations are that, in the absence of data from prospective observational stud- $\frac{0}{2}$ ies or randomised trials, the same criteria should be $x$ used as for starting primary $P$ carinii prophylaxis (see 3 above) ${ }^{10}$ Implicit in these recommendations is the need for close patient monitoring to detect rapidly any fall in 3 CD $4+$ T lymphocyte count or increase in HIV-1 RNA 응 load which identify recurrence of a risk of $P$ carinii pneumonia in individual patients. In this situation $P \frac{D}{2}$ carinii pneumonia must be considered in the differential diagnosis for every patient presenting with respiratory of symptoms.

There are no data to support discontinuation of sec- స్ల

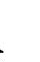


ondary prophylaxis, largely because of the small numbers of such patients in the above mentioned studies ${ }^{58}{ }^{62}$ even though the HIV infected patient with HAART induced partial immune reconstitution might theoretically be at a low risk of $P$ carinii pneumonia. Despite the lack of data, many physicians are now discontinuing secondary prophylaxis. This patient group requires close monitoring if this strategy is followed.

\section{Conclusion}

Prophylaxis of at risk immunosuppressed individuals effectively reduces the attack rate of $P$ carinii pneumonia. Co-trimoxazole is the regimen of first choice for prophylaxis. With long term use of co-trimoxazole for prophylaxis there is evidence for the emergence of resistance in other micro-organisms as well as in $P$ carinii itself. Strategies to limit lifetime exposure to co-trimoxazole are limited by the lack of other equally effective agents. Primary prophylaxis against $P$ carinii pneumonia may safely be discontinued in HIV infected patients with sustained evidence of partial immune reconstitution. These patients require close follow up in order that prophylaxis may be re-started if $\mathrm{CD} 4+\mathrm{T}$ lymphocyte counts fall or HIV-1 RNA load increases.

The spectre of multidrug resistant HIV is looming. In this patient population, and also in the ever increasing population of HIV non-infected immunosuppressed, the need for prophylaxis of $P$ carinii will continue. There is now a pressing need for development of investigational drugs as alternative agents against $P$ carinii and other opportunistic infections.

I am grateful to June Minton, Specialist HIV Pharmacist, UCL Hospitals, London for data on drug costs and to Louise Hinds for typing the manuscript.

1 Hughes WT. Prologue to AIDS. The recognition of infectious opportunists. Medicine 1998;77:227-32.

2 Miller RF. Pneumocystis carinii infection in non-AIDS patients. Curr Opin Infect Dis 1999;12:371-7.

3 Gottlieb MS, Schroff R, Schanker HM, et al. Pneumocystis carini pneumonia and mucosal candidiasis in previously healthy homosexual men. N Engl f Med 1981;305:1425-31.

4 Sepkowitz KA, Brown AE, Armstrong D. Pneumocystis carinii pneumonia without acquired immunodeficiency syndrome: more patients, same risk. Arch Intern Med 1995;155:1125-8.

5 Sepkowitz KA, Brown AE, Telzak EE, et al. Pneumocystis carinii pneumonia among patients without AIDS at a cancer hospital. $\mathcal{F} A M A$ 1992;267:832-7

6 Phair J, Munoz A, Detels R, et al. The risk of Pneumocystis carinii pneumonia among men infected with the human immunodeficiency virus type 1 . N Engl f Med 1990;322:161-5

7 Masur H, Ognibene FP, Yarchoan R, et al. CD4 counts as predictors of opportunistic pneumonias in human immunodeficiency (HIV) infection. Ann Intern Med 1989;111:223-31.

8 Stansell JD, Osmond DH, Charlebois E, et al. Predictors of Pneumocystis carinii pneumonia in HIV-infected persons. Am f Respir Crit Care Med 1997; 155:60-6.

9 Miller RF, Le Noury J, Corbett EL. et al. Pneumocystis carinii infection; current treatment and prevention. $f$ Antimicrob Chemother 1996; 37(Suppl B):33-53.

101999 USPHS/IDSA guidelines for the prevention of opportunistic infections in persons infected with human immunodeficiency virus. infections in persons infect

11 Kulke MH, Vance EH. Pneumocystis carinii pneumonia in patient receiving chemotherapy for breast cancer. Clin Infect Dis 1997;25: $215-8$

12 Walzer PD. Editorial response: Pneumocystis carinii pneumonia in patients without human immunodeficiency virus infection. Clin Infect Dis 1997;25:219-20.

13 Kaplan JE, Hanson DL, Navin TR, et al. Risk factors for primary Pneumocystiscarinii pneumonia in human immunodeficiency virusinfected adolescents and adults in the United States: reassessment of indications for chemoprophylaxis. F Infect Dis 1998;178:1126-32.

14 Sepkowitz KA. Pneumocystis carinii pneumonia without acquired immunodeficiency syndrome: who should receive prophylaxis? Mayo Clin Proc 1996;71:102-3.

15 Miller RF. Prevention and treatment of Pneumocystis carinii pneumonia in patients infected with HIV. Drugs Ther Bull 1994;32:12-15.

16 Ioannidis JPA, Cappelleri JC, Skolnik PR, et al. A meta-analysis of the relataive efficacy and toxicity of Pneumocystis carinii prophylactic regimens. Arch Intern Med 1996;156:177-8.

17 Hardy WD, Feinberg J, Finkelstein DM. et al. A controlled trial of trimethoprim-sulfamethoxazole or aerolised pentamidine for secondary prophylaxis against Pneumocystis carinii pneumonia in patient with the acquired immunodeficiency syndrome. N Engl f Med 1992; 327:1842-8.

18 El-Sadr WM, Murphy RL, McCabe Yurik T, et al. Atovaquone compared with dapsone for the prevention of Pneumocystis carinii pneumonia in patients with HIV infection who cannot tolerate trimethoprim, sulfonamides or both. N Engl f Med 1998;339:1889-95. 9 Machado CM Macedo MCA, Medeiros RSS, et al. Primary Pneumocystis ? carinii pneumonia prophylaxis with aerosolized pentamidine after bone marrow transplantation. Acta Haematol 1998;99:54-6.

20 Bozzette SA, Finkelstein DM, Spector SA, et al. A randomised trial of three anti pneumocystis agents in patients with advanced human immunodeficiency virus infection. N Engl f Med 1995;332:693-9.

21 Schneider MME, Nielsen TL, Nelsing S, et al. Efficacy and toxicity of two doses of trimethoprim-sulfamethoxazole as primary prophylaxis against Pneumocystis carinii pneumonia in patients with human immunodeficiency virus. F Infect Dis 1995;171:1632-6.

22 El-Sadr W, Luskin-Hawk R, Yurik TM, et al. A randomised trail صి

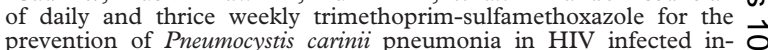
prevention of Pneumocystis carinii pneum
dividuals. Clin Infect Dis 1999;29:775-83.

23 Pitkin AD, Grant AD, Foley NM, et al. Changing patterns of respiratory $\overrightarrow{\vec{\omega}}$ disease in HIV positive patients in a referral centre in the United Kingdom between 1985-7 and 1990-1. Thorax 1993;48:204-7.

24 Jones JJ, Hanson DL, Dworkin MS, et al. Trends in AIDS-related opportunistic infections among men who have sex with men and among injecting drug users, 1991-1996. F Infect Dis 1998;178:114-20.

25 Osmond D, Charlebois E, Lang W, et al. Changes in AIDS survival in time in two San Francisco cohorts of homosexual men, 1983 to 1993. G $7 A M A$ 1994;271:1082-7.

26 Carr A, Swanson C, Penny R, et al. Clinical and laboratory markers of hypersensitivity to trimethoprim-sulfamethoxazole in patients with Pneumocystis carinii pneumonia and AIDS. F Infect Dis 1993;167: $180-5$

27 Koopmans PP, van der Ven AJ, Vree TB, et al. Pathogenesis of hy- is persensitivity reactions to drugs in patients with HIV infection: allergic $\vec{G}$ or toxic? AIDS 1995;9:217-22.

28 Jung AC, Paauw DS. Management of adverse reaction to trimethoprim- 윽 sulfamethoxazole in human immunodeficiency virus-infected patients. Ann Intern Med 1994;154:2402-6.

29 Bachmayer C, Salmon D, Guerun C, et al. Trimethoprim-sulfamethoxazole desensitization in HIV-infected patients: an open study. AIDS 1995;9:299-300.

30 Wilson JM, Guiney DG. Failure of oral trimethoprim-sulfamethoxazole prophylaxis in acute leukemia: isolation of resistant plasmids from $\mathrm{N}$ strains of Enterobacteriaceae causing bacteremia. N Engl F Med 1982; 300:16-20.

31 Slobod KS, Leggiadro RJ. Trimethoprim-sulfamethoxazole-resistant pneumococcal disease in a pediatric acquired immunodeficiency syndrome patient receiving trimethoprim-sulfamethoxazole prophylaxis Pediatr Infect Dis ₹ 1994;13:423-4.

32 Rodriguez-Barradas MC, Tharapel RA, Groover JE, et al. Colonization by Streptococcus pneumoniae among human immunodeficiency virusinfected adults: prevalence of antibiotic resistance, impact of immunization, and charaterization by polymerase chain reaction with BOX primers of isolates from persistent $S$. pneumoniae carriers. $\mathcal{F} \overline{\overline{0}}$
Infect Dis 1997;175:590-7.

33 Martin JN, Rose DA, Hadley WK, et al. Emergence of trimethoprimsulfamethoxazole resistance in the AIDS era. F Infect Dis 1999;180: 1809-18.

34 Dallas WS, Gowen JE, Ray PH, et al. Cloning sequencing, and enhanced expression of the dehydropterate synthase gene of Escherichia coli MC4100. F Bacteriol 1992;174:5961-70.

35 Lopez P, Espinosa M, Greenberg B, et al. Sulfonamide resistance in Streptococcuspneumoniae: DNA sequence of the gene encoding dihydropteroate synthase and characterization of the enzyme. $\mathcal{F}$ Bacteriol 1987;169:4320-6.

36 Triglia T, Menting JG, Wilson C, et al. Mutations in dihydropteroate synthase are responsible for sulfone and sulfonamide resistance in Plasmodium falciparum. Proc Natl Acad Sci USA 1997;94:13944-9.

37 Volpe E, Ballentine SP, Delves CJ. The multifunctional folic acid synthesis fas gene of Pneumocystis carinii encodes dihydroneopterin aldolase, hydroxymethyldihydropterin pyrophosphokinase and dihydropteroate synthase. Eur F Biochem 1993;216:449-58.

38 Mei Q, Gurunathan S, Masur H, et al. Failure of co-trimoxazole in Pneumocystis carinii infection and mutations in dihydropteroate synthase gene. Lancet 1998;351:1631-2.

39 Kazanjian P, Locke AB, Hossler PA, et al. Pneumocystis carinii mutation associated with sulfa and sulfone prophylaxis failures in AIDS patients. AIDS 1998;12:873-8.

40 Meshnick SR. Drug resistant Pneumocystis carinii. Lancet 1999;354. CO 1318-9.

41 Helweg-Larsen J, Benfield TL, Eugen-Olsen J, et al. Effects of mutations in Pneumocystis carinii dihydropteroate synthase gene on outcome of in Pneumocystis carini dihydropteroate synthase gene on outcome

42 Vedantam G, Guay GG, Austria NE, et al. Characterization of mutations क contributing to sulfathiazole resistance in Escherichia coli.Antimicrob Agents Chemother 1998;42:88-93.

$43 \mathrm{Ma} \mathrm{L}$, Borio L, Masur H, et al. Pneumocystis carinii dihydropteroate synthase but not dihydrofolate reductase gene mutations correlate with prior trimethoprim-sulfamethoxazole or dapsone use. F Infect Dis 1999;180:1969-78

44 Walzer PD, Kim CK, Foy JM, et al. Inhibitors of folic acid synthesis in the treatment of experimental Pneumocystis carinii pneumonia. Antimicrob Agents Chemother 1988;32:96-103.

45 Kunz S, Junker U, Blaser J, et al. The SCID mouse as an experimental model for the evaluation of anti-Pneumocystis carinii therapy. 7 Antimicrob Chemother 1995;36:137-55.

46 Walker DJ, Wakefield AE, Dohn MN, et al. Sequence polymorphisms in the Pneumocystis carinii cytochrome $b$ gene and their association

47 Marreros E, Martinez CM, Almela MJ, et al. Sordarins, in vitro activities of new antifungal derivatives against pathogenic yeasts, Pneumocystis carinii and filamentous fungi. Antimicrob Agents Chemother 1998;42: 2863-9.

48 Hughes WT, Killmar JT. Synergistic anti-Pneumocystis carinii effects of 
erythromycin and sulfisoxazole. F Acq Immune Defic Syndr 1991;4: $532-7$

49 Dunne MW, Bozette S, McCutchan JA, et al. Efficacy of azithromycin in prevention of Pneumocystis carinii pneumonia: a randomised trial

50 Jorde UP, Horowitz HW, Wormser GP. Significance of drug interaction with rifampicin in Pneumocystis carinii pneumonia prophylaxis. Arch Intern Med 1992;152:43-8.

51 Pozniak AL, Miller R, Ormerod LP. The treatment of tuberculosis in HIV-infected persons. AIDS 1999;13:435-45.

52 Hammer SM, Squires KE, Hughes MD, et al. A controlled trial of two nucleoside analogues plus indinavir in persons with human immunodeficiency virus infection and CD4 counts of 200 per cubic millimetre or less. N Engl F Med 1997;337:725-33.

53 Brodt HR, Kamp SBS, Gute P, et al. Changing incidence of AIDSdefining illnesses in the era of antiretroviral combination therapy. AIDS 1997;11:1731-8.

54 Jacobson MA, French $M$. Altered natural history of AIDS-related opportunistic infections in the era of patent combination antiretroviral opportunistic infections in the era of patent

55 Autran B, Carcelain G, Li TS, et al. Positive effects of combined antiretroviral therapy on CD $4+\mathrm{T}$ cell homeostasis and function in advanced HIV disease. Science 1997;277:112-6.

56 Komanduri KV, Viswanathan MN, Wieder ED, et al. Restoration of cytomegalovirus-specific CD4 + T-lymphocyte responses after ganciclovir and highly active antiretroviral therapy in individuals infected with HIV-1. Nature Med 1998;4:953-6.

$57 \mathrm{Li}$ TS, Tubiana R, Katlama C, et al. Long-lasting recovery in CD4 Tcell function and viral load reduction after highly active antiretroviral therapy in advanced HIV-1 disease. Lancet 1998;351:1682-6.

58 Weverling GJ, Mocroft A, Ledergerber B, et al. Discontinuation of Pneumocystis carinii pneumonia prophylaxis after start of highly active antiretroviral therapy in HIV-1 infection. Lancet 1999;353:1293-8.

59 Furrer H, Egger M, Opravil M. et al. Discontinuation of primary prophylaxis against Pneumocystiscarinii pneumonia in HIV-1 infected adults treated with combination antiretroviral therapy. $N$ Engl f Med 1999;340:1301-6.

60 Schneider MME, Borleffs JCC, Stolk RP, et al. Discontinuation of prophylaxis for Pneumocystis carinii pneumonia in HIV-1 infected patients treated with highly active antiretroviral therapy. Lancet 1999; 353:201-3.

61 Kirk O, Lundgren JD, Pedersen C, et al. Can chemoprophylaxis against opportunistic infections be discontinued after an increase in CD4 cells induced by highly active antiretroviral therapy? AIDS 1999;13: $1647-51$.

62 Rodriguez-Guardado A, Maradona JA, Carton JA, et al. Pneumocystis carinii prophylaxis can be discontinued after CD 4 + cell recovery over $200 \times 1 \phi$ L. AIDS 1998;12:2355-6. 